\title{
Inhalt *
}

Entscheidungen des Staatsgerichtshofs für das Land Baden-Württemberg . . . . Seite

Nr. 1 8.2.2000 Rechts- und Gesetzeskraft eines NormenkontrollGR 1/98 Urteils, das die Unvereinbarkeit einer Norm mit der Landesverfassung feststellt ..........

Nr. 2 8.2.2000 Kein Normenkontrollverfahren gegen Landesgesetze GR 1/00 auf Antrag eines Einzelnen und keine Landesverfassungsbeschwerde vor dem Staatsgerichtshof für das Land Baden-Württemberg ...........

Nr. 3 19.5.2000 Möglichkeit der Verletzung von Rechten einer LandGR 2/99 tagsfraktion oder des Landtags durch Regierungsmitglieder oder die Landesregierung infolge Zuwiderhandlungen gegen Art. 53 Abs. 2 Sätze 1, 2 LV ...

Nr. 4 5.12.2000 Verfahrensbeendende Wirkung einer AntragsrückGR 3/99 nahme .................

Entscheidungen des Verfassungsgerichtshofs des Landes Berlin

Nr. 1 21.2.2000 Wahlprüfungsverfahren; Einreichung von WahlvorVerfGH schlägen; Begriff der politischen Partei . . . . . . $122 / 99$

Nr. $2 \quad 8.3 .2000$

Überprüfung einer gerichtlichen Entscheidung am VerfGH $121 / 98$ Maßstab des Grundrechts auf rechtliches Gehör; Wiedereinsetzung in den vorigen Stand; Wartezeit bei angezeigter Verspätung eines Verfahrensbeteiligten .

Nr. $3 \quad 18.5 .2000$

Einspruch gegen die Feststellung des NichtzustandeVerfGH $78 / 99$ kommens eines Volksbegehrens; Anforderungen an das Abstimmungsverfahren ...........

Nr. $4 \quad 15.6 .2000$

Beteiligtenfähigkeit der Berliner Bezirke im OrganVerfGH 47/99 streit- und Verfassungsbeschwerdeverfahren . . . .

* Die entsprechend gekennzeichneten Entscheidungen sind entweder nur mit den Leitsätzen abgedruckt oder gekürzt wiedergegeben und im Volltext bei den jeweiligen Landesverfassungsgerichten erhältlich (Adressen s. Anhang). 
Nr. $5 \quad 23.11 .2000$ VerfGH $117 / 99$

Nr. 6

23.11.2000 VerfGH $72 / 00$

Nr. $7 \quad 21.12 .2000$ VerfGH $136 / 00$
Verfassungsbeschwerde gegen Akte im Rahmen des Wahlverfahrens; Verhältnis von Wahlprüfung und Verfassungsbeschwerde; Geltendmachung einer Verletzung des subjektiven Wahlrechts (hier: Bereitstellung von Wahlschablonen für blinde Wähler); Rechtsschutzbedürfnis . . . . . . . . .

Überprüfung einer zivilgerichtlichen Entscheidung am Grundrecht auf Eigentum; Mieterhöhung nach Erhalt öffentlicher Fördermittel für Modernisierungsmaßnahmen; Ansatz von Kürzungsbeträgen . . .

Parteifähigkeit der Studentenschaft einer Hochschule im Verfassungsbeschwerdeverfahren . . . . . . .

Entscheidungen des Verfassungsgerichts des Landes Brandenburg

Nr. ${ }^{*} \quad 20.1 .2000$ VfGBbg

$53 / 98$

$3 / 99$

Nr. $2^{*} \quad 17.2 .2000$ VfGBbg $39 / 99$

Nr. 3* 17.2 .2000 VfGBbg $43 / 99$

Nr. 4* 17.2.2000 VfGBbg $45 / 99$

Nr. 5* 16.3 .2000 VfGBbg $42 / 99$
Kommunale Selbstverwaltung und Gesetz zur rechtlichen Stabilisierung der Zweckverbände für Wasser-

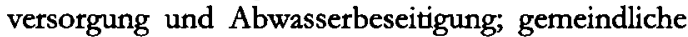
Organisationshoheit und rückwirkende Heilung fehlerhafter Zweckverbandsgründungen $\ldots \ldots$.

Verstoß gegen das Willkürverbot und das Recht auf rechtliches Gehör und Recht auf ein faires Verfahren bei Widerruf einer Strafaussetzung zur Bewährung trotz Unklarheit über Mitteilung des Auflagenbeschlusses $\ldots \ldots \ldots \ldots \ldots \ldots$

Keine Verletzung von Prozeßgrundrechten nach unterbliebener Vollziehung einer einstweiligen Verfügung im Wege der Auslandszustellung, wenn der Verfügungskläger sich nicht nach dem Verbleib des $\mathrm{Zu}$ stellungsnachweises erkundigt $\ldots \ldots \ldots \ldots$

Pflicht zur unverzüglichen Benachrichtigung einer Person des Vertrauens bei Entscheidung über den Freiheitsentzug für den Fall, daß das Vertrauensverhältnis zu dem Pflichtverteidiger gestört ist . . . . . .

Keine Verletzung von Prozeßgrundrechten bei Berücksichtigung veränderter Anträge im Verfahren auf $\mathrm{Erla} ß$ einer einstweiligen Verfügung wegen presserechtlicher Gegendarstellung . . . . . . . . . . . 
Nr. 6* 16.3.2000 VfGBbg $6 / 00$

Nr. ** $^{*} \quad 16.3 .2000$ VfGBbg $2 / 00$

Nr. 8* 15.6.2000 VfGBbg 32/99
Zur Frage einer Verletzung von Prozeßgrundrechten in einem zivilrechtlichen Verfahren für den Fall, daß das einfache Bestreiten wegen vorangegangenen Prozeßverhaltens der Partei nicht berücksichtigt wird; Bedeutung eines Hinweisbeschlusses $\ldots . .$.

Anforderungen an das Verfahren im Räumungsprozeß wegen Eigenbedarfs $\ldots . . . \ldots$.

Kommunale Verfassungsbeschwerde gegen die Verordnung zur Verbindlicherklärung des Braunkohlenplans Tagebau Jänschwalde; Abgrenzung zwischen „Auflösung" einer Gemeinde und (anderweitigem) Eingriff in die Planungshoheit; Erforderlichkeit einer eigenen Entscheidungsverantwortlichkeit des Verordnungsgebers; Erforderlichkeit gesetzlicher Vorgaben für die Zusammensetzung und Arbeitsweise des Braunkohlenausschusses ............

Keine generelle Verpflichtung des Gerichts (hier: im Verkehrsunfallprozeß) zur Einholung eines Zweitgutachtens (hier: zur Ermittlung der Ausgangsgeschwindigkeit) $\ldots \ldots \ldots \ldots \ldots$

Nr. 10* 12.10 .2000 VfGBbg $19 / 00$

Wahlprüfungsbeschwerde: Beschwerdeberechtigung, Beginn der Beschwerdefrist, Anforderungen an die Rechtsmittelbelehrung; kein Nachrücken einer Ersatzperson aus der Landesliste, wenn ausscheidender Abgeordneter im Wahlkreis gewählt worden ist und die Partei, der er angehört, über bis zu zwei Überhangmandate verfügt

Bestimmtheitsgebot und gesetzlicher Biotopschutz; Bedeutung eines landesrechtlichen Biotop-Verzeichnisses

Verletzung des Rechts auf Gelegenheit zur Zuziehung eines Rechtsbeistands vor richterlicher Entscheidung über Freiheitsentziehung bei nicht rechtzeitiger Ankündigung einer Anhörung vor der Strafvollstreckungskammer $\ldots \ldots \ldots \ldots$

Nr. 13* 16.11 .2000 VfGBbg $31 / 00$
Zur Pflicht der Landesregierung zur Beantwortung parlamentarischer Anfragen ,nach bestem Wissen und Gewissen" ................. 
Nr. 14* 16.11.2000

VfGBbg

Subsidiarität der Verfassungsbeschwerde gegenüber VfGBbg $49 / 00$ fachgerichtlichem Hauptsacheverfahren bei Untersagung einer Äußerung durch einstweilige Verfügung

Entscheidungen des Staatsgerichtshofs der Freien Hansestadt Bremen

Nr. $1 \quad 14.2 .2000 \quad$ Volksbegehren zur Verfassungsänderung $\ldots \ldots$ St $1 / 98$

Nr. $2 \quad 29.8 .2000$

Kommunalwahlrecht und Stadtstaatlichkeit $\ldots$. 199 St 4/99

Entscheidungen des Hamburgischen Verfassungsgerichts (in diesem Band keine Entscheidungsveröffentlichung)

Entscheidungen des Staatsgerichtshofs des Landes Hessen

Nr. $1 \quad 5.4 .2000$

Rechtliches Gehör und gerichtlicher Hinweis . . .

P.St. 1302

Nr. $2 \quad 14.6 .2000$

Prüfungsgegenstand und Frist bei Grundrechtsklage

P.St. 1351

Nr. $3 \quad 9.8 .2000$

Rechtliches Gehör; Wahlprüfung

P.St. 1547

Nr. $4 \quad$ 14.9. 2000

Steuererhebungsermächtigung von Landkreisen und P.St. 1314 kreisfreien Städten - Jagdsteuer

Nr. $5 \quad$ 6.12.2000 Staatsgerichtshof - Ausscheiden eines berufsrichterP.St. 1596 lichen Mitgliedes . . . . . . . . . . . .

Entscheidungen des Landesverfassungsgerichts Mecklenburg-Vorpommern

Nr. $1 \quad 18.5 .2000$

Dynamische Verweisung; Überwachung von WohLVerfG $5 / 98$ nungen zum Zweck der Gefahrenabwehr; vorbeugende Bekämpfung von Straftaten im Sinne der polizeilichen Aufgabenzuweisung; organisierte Kriminalität; besondere Vertrauensverhältnisse; Amts- und Berufsgeheimnis; Umwidmung personenbezogener Daten zur Strafverfolgung $\ldots \ldots \ldots \ldots \ldots$ 
Nr. 2 14.12.2000 Beteiligtenfähigkeit politischer Parteien im OrganLVerfG 4/99 streitverfahren; Wettbewerbschancen einer Partei als Bestandteil des verfassungstechtlichen Status; Recht auf Teilhabe am Verfassungsleben; 5\%-Sperrklausel; gesetzgeberisches Unterlassen; mögliche Gefährdung der Funktionsfähigkeit einer Kommunalvertretung .

Entscheidungen des Niedersächsischen Staatsgerichtshofs

Nr. 1 24.2.2000 Wahlprüfung; Bedeutung der Wahlkreisgröße für die StGH 2/99 Wahlrechtsgleichheit; Bestandsschutz einer gewählten Volksvertretung .............

Entscheidungen des Verfassungsgerichtshofes des Saarlandes (in diesem Band keine Entscheidungsveröffentlichung)

Entscheidungen des Verfassungsgerichtshofes des Freistaates Sachsen

Nr. 1 24.2.2000 Eigentumsgarantie; Enteignung; vorzeitige BesitzVf. 37-IV-99 einweisung zum Zweck der Energieversorgung; Gesetzesvorbehalt; erforderliche gesetzliche Entschädigungsgrundlage $\ldots \ldots \ldots \ldots \ldots$

Nr. $2 \quad 9.3 .2000$

Organstreitverfahren; vom Präsidium des Sächsischen Vf. 3-I-00

Vf. 4-I-00 Landtags erlassene Ausführungsbestimmungen zu $₫ 6$ Abs. 4 SächsAbgG unzulässiger Verfahrensgegenstand; zum Antragsgegner $\ldots . . . \ldots . .$.

Heuersdorfgesetz; kommunale Selbstverwaltungsgarantie; Freigabe eines Gemeindegebiets zur Devastierung; verfassungstechtliche Anforderungen an eine Anhörung nach Art. 88 Abs. 2 S. 3 SächsVerf; zum Einschätzungs- und Prognosevorrang des Gesetzgebers ................

Nr. $4 \quad 17.8 .2000$ Vf. 62-II-99

Abstrakte Normenkontrolle; Gesetz zur Neuordnung der öffentlich-rechtlichen Kreditinstitute im Freistaat Sachsen einschließlich der Sächsischen Aufbaubank GmbH vom 3.5.1999; Selbstverwaltungsgarantie; kommunale Sparkasse als Angelegenheit der örtlichen Gemeinschaft; Durchbrechung des objektiv-rechtlichen Prinzips dezentraler Aufgabenverteilung; Gesetzesvorbehalt gem. Art. 82 Abs. 2 SächsVerf; 
Rechtfertigung durch überwiegende Allgemeinwohlgründe; Einschätzungs- und Prognosevorrang; verfassungskonforme Auslegung

Entscheidungen des Landesverfassungsgerichts Sachsen-Anhalt

Nr. $1 \quad$ 13.7.2000 Kommunaler Finanzausgleich - Kostendeckung bei LVG $20 / 97$ Aufgabenübertragung $\ldots \ldots \ldots \ldots \ldots$

Nr. 2 29.8.2000 Volksinitiative - Landtagsbehandlung des GesetzentLVG $1 / 00 \quad$ wurfes $\ldots \ldots \ldots \ldots \ldots \ldots$

Entscheidungen des Thüringer Verfassungsgerichtshofes

Nr. $1 \quad 25.5 .2000 \quad$ Verfahren der abstrakten Normenkontrolle betreffend VerfGH $₫ 8$ des Thüringer Abgeordnetenüberprüfungsgesetzes 2/99 (Mandatsverlust wegen Zusammenarbeit mit dem MfS/AfNS) $\ldots \ldots \ldots \ldots \ldots \ldots \ldots$

Nr. 2* 25.5.2000 Organstreitverfahren betreffend den Beschluß des VerfGH Thüringer Landtags über den Mandatsverlust einer $4 / 99$ Abgeordneten ...............

Nr. 3* 25.5.2000 Antrag auf Erlaß einer einstweiligen Anordnung VerfGH VerfGH 6/00 betreffend den Beschluß eines Land6/00 tagsausschusses über den Ausschluß eines Fraktionsmitarbeiters von den nichtöffentlichen Ausschuß-

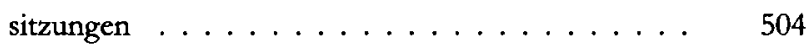

Sachregister $\quad \ldots \ldots \ldots \ldots \ldots \ldots \ldots \ldots \ldots \ldots$

Gesetzestegister $\quad \ldots \ldots \ldots \ldots \ldots \ldots \ldots \ldots \ldots \ldots \ldots$

Verzeichnis der Verfassungsgerichte der Länder . . . . . . . . . . . 541 